\title{
Estudo por Imasem da Articulação Carpometacarpal do Polegar
}

\section{Imasing Study of the Carpometacarpal Joint of the Thumb}

\author{
Claudia Kazue Yamaguchi ${ }^{(1)}$, Maria Carolina Guimarães ${ }^{(2)}$, Fernando Santos Emerich Gomes ${ }^{(1)}$, André Yui
} Aihara $^{(2)}$, Cassiano Leão Bannwart ${ }^{(3)}$, Ivan Chakkour ${ }^{(3)}$, Jamil Natour ${ }^{(4)}$, Artur da Rocha Correa Fernandes ${ }^{(2)}$

\section{INTRODUÇÃO}

O polegar é responsável por, aproximadamente, $60 \%$ da função de preensão da mão, o que pode ser atribuído à sua posição única e à movimentação multiplanar da articulação carpometacarpal, que é formada pela faceta proximal do primeiro metacarpal (MTC) e pela faceta distal do trapézio. Quando a dor ou a instabilidade estão presentes, as atividades simples do dia-a-dia podem ser comprometidas ${ }^{(1)}$.

$\mathrm{Na}$ investigação da dor no polegar, deve-se sempre considerar a osteoartrite, que tem três causas principais: inflamatória, traumática e idiopática. Nesta última, temse maioria absoluta formada por mulheres no período pós-menopausa, que apresentam dor nessa articulação, de maneira insidiosa ${ }^{(2)}$.

O objetivo deste estudo é demonstrar por meio da radiografia simples (RX) e da ressonância magnética ( $\mathrm{RM})$, a anatomia óssea e capsular e as afecções que envolvem a articulação carpometacarpal do polegar.

\section{ANATOMIA}

As facetas proximal do primeiro MTC e distal do trapézio, que formam a articulação carpometacarpal do polegar, tem forma de sela ${ }^{(3)}$. Há um complexo ligamentar que estabiliza o trapézio e a articulação carpometacarpal do polegar que consiste de 16 ligamentos, sendo quatro os principais $^{(4)}$ (Figura 1):

- oblíquo anterior que é uma banda curta e espessa de fibras que se estendem do tubérculo palmar do trapézio até a base do primeiro MTC (Figura 2);

- dorsal radial que se origina do aspecto dorso-radial do trapézio e se insere na base do primeiro MTC, esse ligamento é reforçado pelo tendão do abdutor longo do polegar (TALP);

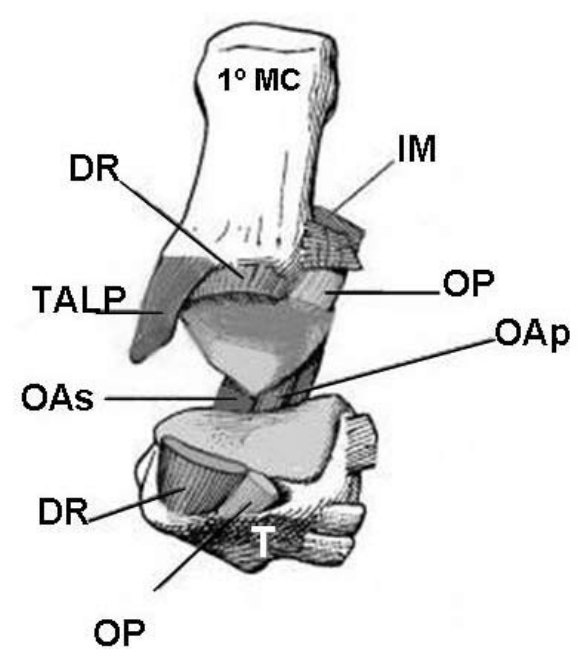

Figura 1 - Desenho esquemático da articulação carpometacarpal aberta (modificado a partir da referência 4). $0 \mathrm{As}=$ oblíquo anterior, porção superficial; $\mathrm{A} 0 \mathrm{p}=$ oblíquo anterior, porção profunda; $\mathrm{DR}=$ dorsal radial; $\mathrm{OP}=$ oblíquo posterior; $\mathrm{IM}=$ intermetacarpal; $\mathrm{T}=$ trapézio; $1^{\circ} \mathrm{MC}=$ primeiro metacarpal.

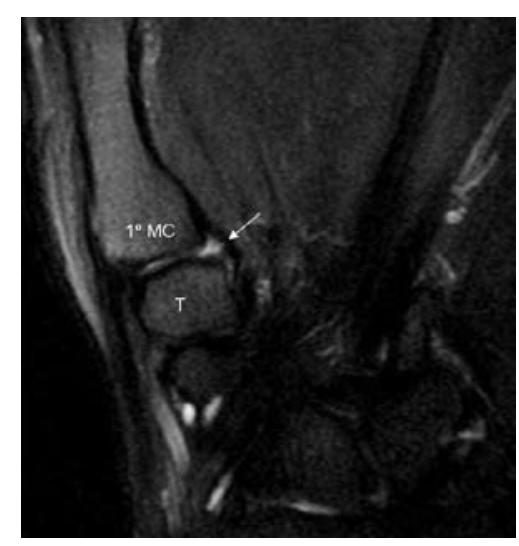

Figura 2 - Imagem de RM no plano coronal, seqüência ponderada em T2 com saturação do sinal de gordura - o ligamento oblíquo anterior (seta) é visto como uma banda de baixo sinal estendendo-se da face ventral do trapézio $(\mathrm{T})$ à base do primeiro metacarpal ( $\left.1^{\circ} \mathrm{MC}\right)$.

1. Serviço de Diagnóstico por Imagem da Santa Casa de Misericórdia de São Paulo.

2. Departamento de Diagnóstico por Imagem da Universidade Federal de São Paulo (UNIFESP).

3. Departamento de Ortopedia e Traumatologia da Santa Casa de Misericórdia de São Paulo.

4. Disciplina de Reumatologia da UNIFESP

Endereço para correspondência: Artur R. C. Fernandes, DDI/UNIFESP, Rua Botucatu, 740, 04023-900, São Paulo, SP, e-mail: artur_personal@yahoo.com.br 
- oblíquo posterior que se estende do tubérculo ulnar dorsal do trapézio até o tubérculo ulnar da base do primeiro MTC;

- intermetacarpal (IM) que se estende da base do segundo MTC até o aspecto ulnar da base do primeiro $\mathrm{MTC}^{(1,5)}$.

\section{AVALIAÇÃO RADIOGRÁFICA}

Geralmente são realizadas RX em AP e perfil da mão.

Em um estudo mais específico da articulação carpometacarpal do polegar, pode-se incluir as incidências perfil verdadeiro (Figura 3), póstero-anterior (Figura 4) e hiperpronada ântero-posterior de Robert (Figura 5), que possibilitam melhor avaliação dos espaços e superfícies articulares e das relações entre o trapézio e o primeiro MTC e entre o trapézio e o escafóide ${ }^{(1)}$.
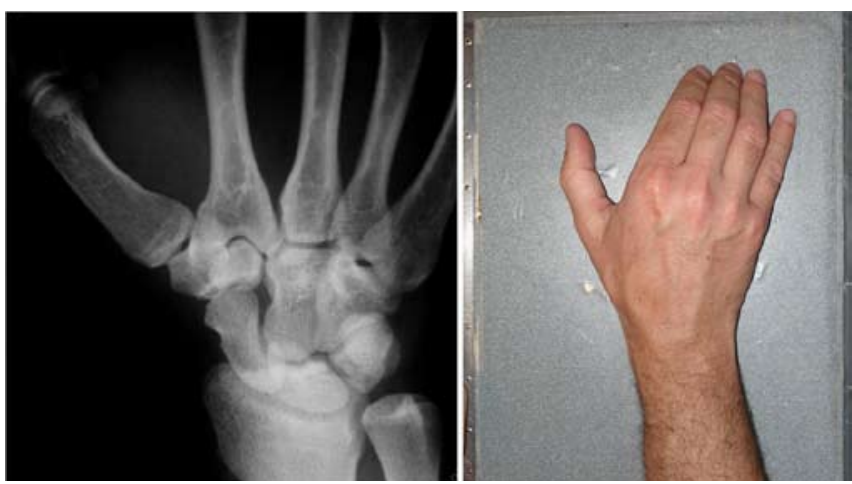

Figura 3 - Incidência perfil verdadeiro, demonstrada pela radiografia e foto com posição correta da realização do exame.
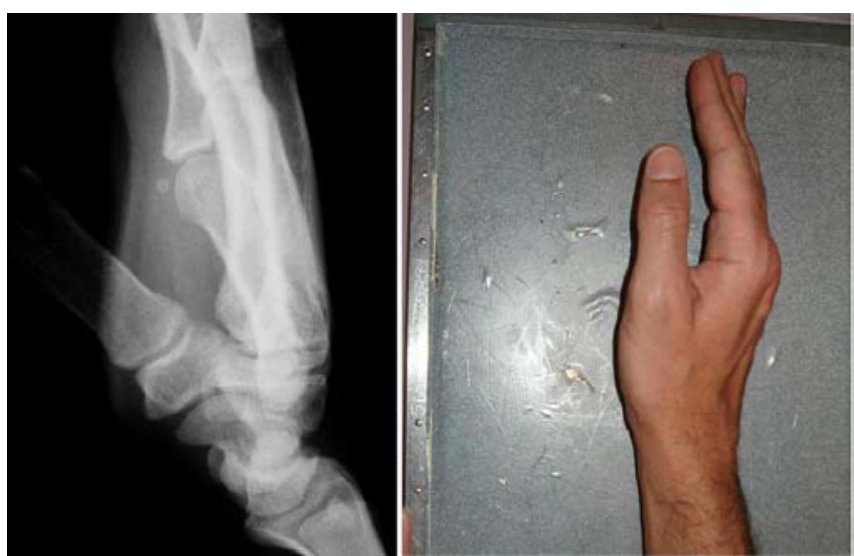

Figura 4 - Incidência póstero-anterior, demonstrada pela radiografia e foto com posição correta da realização do exame.

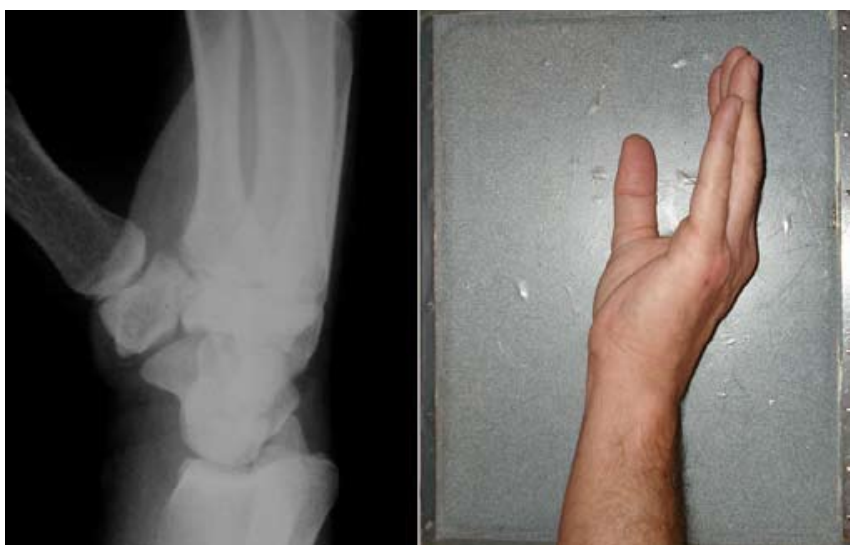

Figura 5 - Incidência hiperpronada ântero-posterior de Robert, demonstrada pela radiografia e foto com posição correta da realização do exame.

\section{AVALIAÇÃO PELA RM}

A RM permite a caracterização dos principais ligamentos da articulação carpometacarpal e a avaliação da integridade ligamentar, além de detecção das lesões condrais e fraturas ocultas.

\section{OSTEOARTRITE}

A prevalência da artrose, osteoartrose ou osteoartrite carpometacarpal do polegar aumenta com a idade e há predomínio em mulheres após a menopausa. A evidência radiográfica de artrose está presente em um terço destas mulheres, porém somente um terço dessas é sintomática. Postula-se que a articulação seja menor e menos congruente nas mulheres e que a cartilagem hialina seja mais fina, predispondo maior incidência de osteoartrite. Uma combinação de fatores parece ser responsável pela degeneração articular, incluindo alto estresse local, incongruência articular e frouxidão ligamentar ${ }^{(1)}$.

Apesar de haver controvérsias, acredita-se que o ligamento oblíquo anterior é o estabilizador primário da carpometacarpal do polegar. A perda deste suporte ligamentar resulta em hipermobilidade e aumento de forças de tensão na articulação e parece ser causa da artropatia degenerativa ${ }^{(6)}$.

\section{CLASSIFICAÇÃO RADIOLÓGICA}

A classificação de Eaton e Littler é a mais comumente utilizada na avaliação radiológica da osteoartrite do primeiro carpometacarpal, visando a escolha do tratamento ${ }^{(1)}$ :

- estágio I - contorno articular normal, o espaço pode estar alargado por sinovite secundária, subluxação pode estar presente;

- estágio II - leve redução do espaço articular e esclerose com osteófitos menores que $2 \mathrm{~mm}$ (Figura 6); 


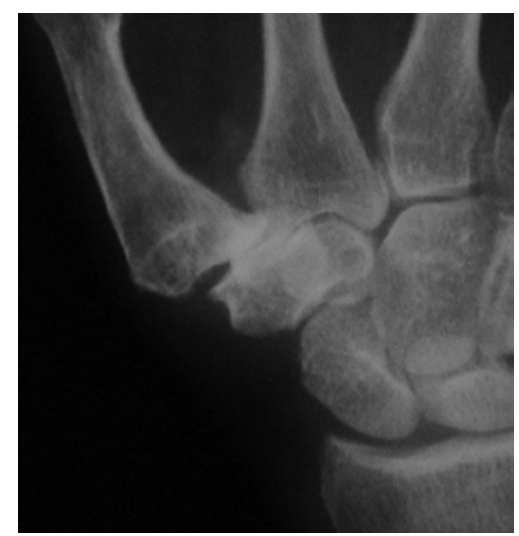

Figura 6 - Estágio II da artrose, segundo Eaton e Litter, caracterizado pela pequena redução do espaço articular associada à esclerose e osteófitos menores que $2 \mathrm{~mm}$.

- estágio III - redução significativa do espaço articular e esclerose com osteófitos marginais maiores que $2 \mathrm{~mm}$; articulação escafotrapezoidal normal (Figura 7);

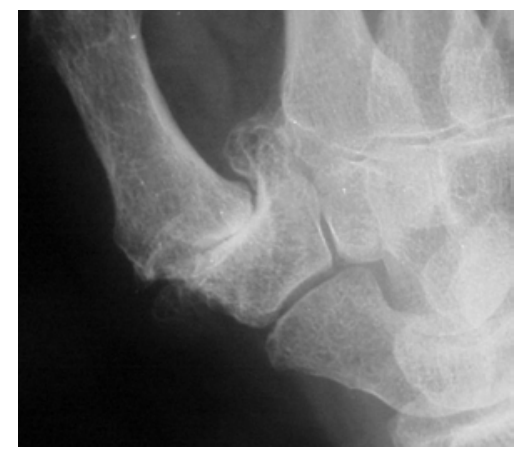

Figura 7 - Estágio III da artrose, segundo Eaton e Litter, caracterizado por redução significativa do espaço articular associada à esclerose e osteófitos marginais maiores que $2 \mathrm{~mm}$; articulação escafotrapezoidal normal.

- estágio IV - igual ao III, acrescido de artrose escafotrapezoidal $^{(1)}$ (Figura 8).

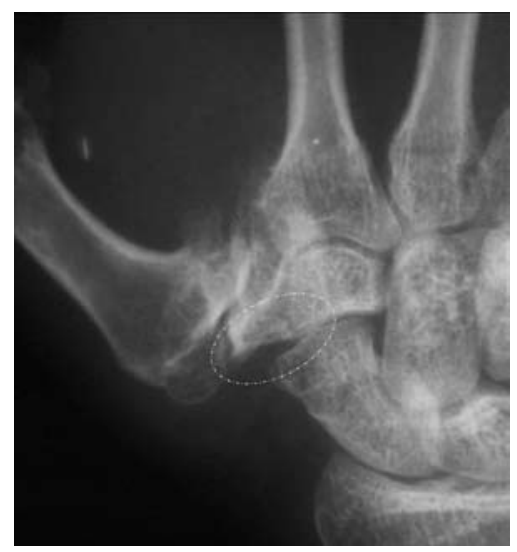

Figura 8 - Estágio IV da artrose, segundo Eaton e Litter, com comprometimento das articulações carpometacarpal e escafotrapezoidal (círculo).

\section{LESÃO LIGAMENTAR CRÔNICA}

Nas lesões traumáticas agudas, o ligamento oblíquo anterior (Figura 9) é o mais comumente lesado, em geral na sua inserção na base do metacarpal, distal à margem articular, seguido pelo radial dorsal, cujas lesões ocorrem na sua inserção no trapézio ${ }^{(5)}$.

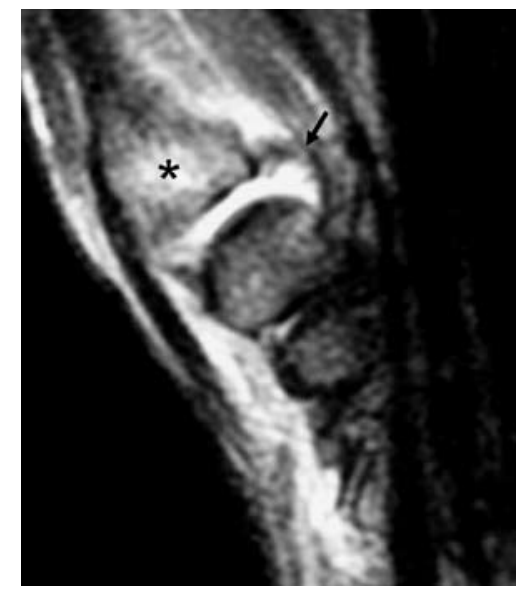

Figura 9 - Paciente do sexo feminino, 41 anos, com história de acidente de esqui há um mês e dor no polegar - imagem de RM no plano coronal, seqüência ponderada em T2 com saturação do sinal de gordura demonstra edema medular da base do primeiro MTC $\left(^{*}\right)$ e de partes moles periarticulares, e sinais de lesão parcial do ligamento oblíquo anterior caracterizada por espessamento, aumento do sinal e irregularidade das fibras distais.

Cronicamente, quando o trauma leva à instabilidade e não é tratado adequadamente, pode determinar artrose secundária, necessitando, assim, de reconhecimento precoce. Nas lesões crônicas, a RM permite detectar frouxidão e espessamento ligamentar e também podem ser identificados ganglions, além da osteoartrite ${ }^{(5)}$ (Figura 10).

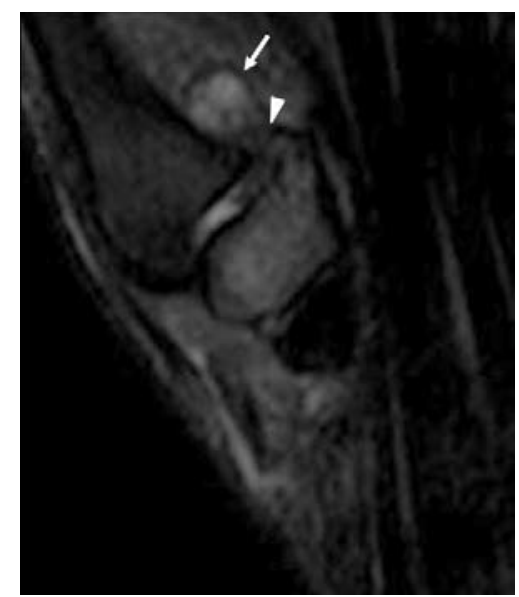

Figura 10 - Paciente do sexo feminino, 40 anos, trauma com fratura do trapézio há dois anos, atualmente com dor na base do polegar - imagem de RM no plano coronal, seqüência ponderada em T2 com saturação do sinal de gordura demonstra edema medular do trapézio e sinais de lesão crônica da porção distal do ligamento oblíquo anterior (cabeça de seta), associados a ganglion cístico (seta). 


\section{OUTRAS ARTROPATIAS}

A articulação carpometacarpal do polegar também pode ser comprometida por artropatias inflamatórias (Figura 11) e doenças de depósito (Figura 12). Estas artropatias têm achados clínicos e radiológicos próprios, que auxiliam no diagnóstico diferencial ${ }^{(7)}$.

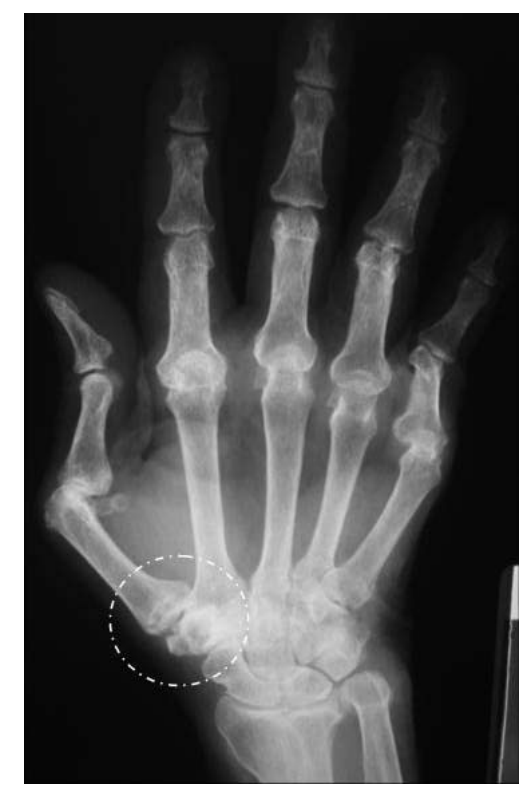

Figura 11 - Artrite reumatóide (RX) - comprometimento das articulações do punho e das metacarpofalângicas, além de envolvimento da carpometacarpal do polegar (círculo).

\section{TRATAMENTO}

O tratamento das lesões ligamentares agudas carpometacarpais pode ser conservador com imobilização por órtese, por cerca de quatro semanas e uso de antiinflamatórios.

\section{REFERÊNCIAS}

1. Young SD, et al.: Thumb carpometacarpal artrhosis. Journal of the American Society for Surgery of the Hand 4: 73-94, 2004.

2. Rezende $M R$, et al.: Nova técnica cirúrgica para o tratamento da artrite trapezometacarpiana. Rev Bras Ortop 33(12): 982-6, 1998.

3. Kauer JM: Functional anatomy of the carpometacarpal joint of the thumb. Clin Orthop Relat Res 220: 7-13, 1987.

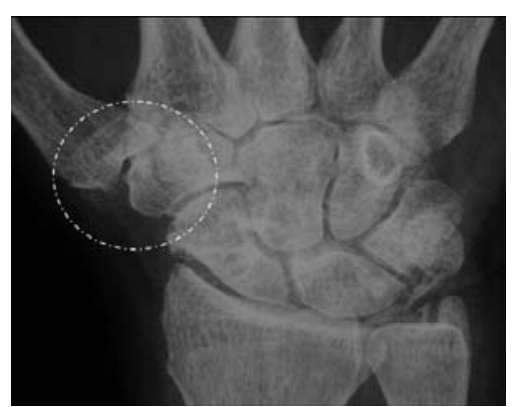

Figura 12 - Artropatia microcristalina (RX) - condrocalcinose na face ulnar do punho, na articulação radiocarpal e envolvimento da carpometacarpal do polegar (círculo).

A cirurgia pode ser realizada em casos de artrose estabelecida. A artrodese carpometacarpal pode ser realizada em pacientes mais jovens e ativos, com artrose nos estágios II e III de Eaton e Littler. A trapezectomia isolada é realizada em pacientes muito idosos, que não requerem força ou função, com artrose nos estágios III e IV de Eaton e Littler. A trapezectomia associada a suspensoplastia pode ser indicada para todos os estágios da artrose, exceto o estágio I. A excisão completa do trapézio é feita rotineiramente porque trata também artrose escafotrapezoidal não-diagnosticada. A suspensoplastia consiste da interposição e do reforço da articulação pelo tendão flexor radial do carpo ${ }^{(1)}$.

\section{CONCLUSÕES}

O estudo radiográfico da articulação carpometacarpal possibilita a avaliação da congruência articular e de lesões ósseas, como fraturas/avulsões, além de detectar e estadiar a artropatia degenerativa.

A RM permite a avaliação da integridade ligamentar, das lesões condrais e fraturas ocultas.

4. Bettinger PC, et al.: Functional ligamentous anatomy of the trapezium and trapeziometacarpal joint (gross and arthroscopic). Hand Clinics 17(2): 151-68, 2001.

5. Conell DA, et al.: MR imaging of thumb carpometacarpal jont ligament injuries. Journal of Hand Surgery 29B: 46-54, 2004.

6. Imaeda $\mathrm{T}$, et al.: Anatomy of trapeziometacarpal ligaments. Journal of Hand Surgery 18A: 226-31, 1993.

7. Resnick D: Diagnosis of bone and joint disorders. $3^{\mathrm{a}} \mathrm{ed}$. Buenos Aires: Editorial Médica Panamericana, 1984. 